\title{
LA-UR-00: 4892
}

Approved for public release;

distribution is unlimited

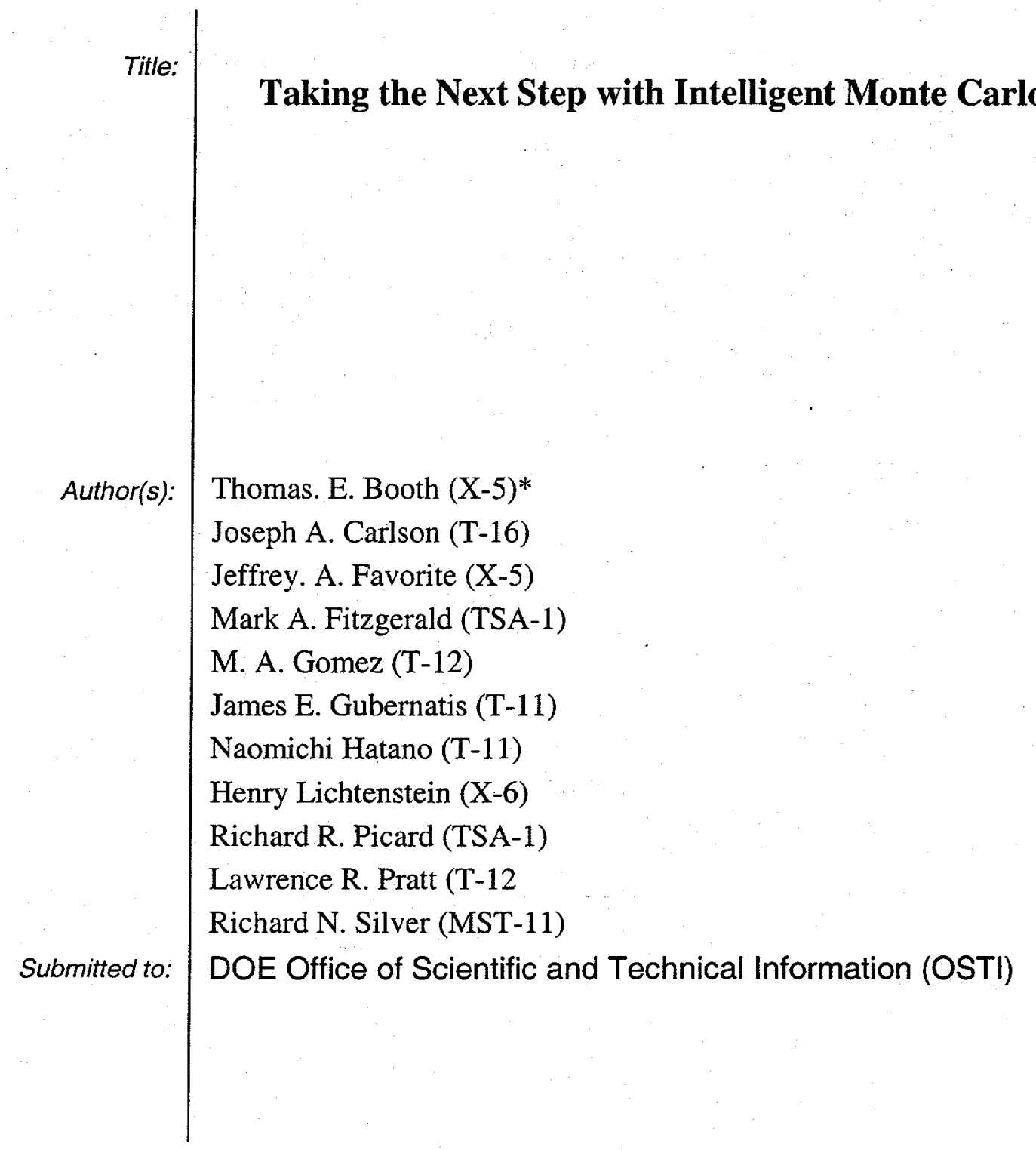

Los Alamos National Laboratory, an affirmative actionvequal opportunity empioyer, is operated by the University of California for the U.S. Department of Energy under contract W-7405-ENG-36. By acceptance of this article, the publisher recognizes that the U.S. Government retains a nonexclusive, royaltyfree license to publish or reproduce the published form of this contribution, or to allow others to do so, for U.S. Government purposes. Los Alamos National Laboratory requests that the publisher identify this article as work performed under the auspices of the U.S. Department of Energy. Los Alamos National Laboratory strongly supports academic freedom and a researcher's right to publish; as an institution, however, the Laboratory does not endorse the viewpoint of a publication or guarantee its technical correctness. 


\section{DISCLAIMER}

This report was prepared as an account of work sponsored by an agency of the United States Government. Neither the United States Government nor any agency thereof, nor any of their employees, make any warranty, express or implied, or assumes any legal liability or responsibility for the accuracy, completeness, or usefulness of any information, apparatus, product, or process disclosed, or represents that its use would not infringe privately owned rights. Reference herein to any specific commercial product, process, or service by trade name, trademark, manufacturer, or otherwise does not necessarily constitute or imply its endorsement, recommendation, or favoring by the United States Government or any agency thereof. The views and opinions of authors expressed herein do not necessarily state or reflect those of the United States Government or any agency thereof. 


\section{DISCLAIMER}

Portions of this document may be illegible in electronic image products. Images are produced from the best available original document. 


\title{
Taking the Next Step with Intelligent Monte Carlo
}

Thomas E. Booth* (X-5), Joseph A. Carlson (T-16), Jeffrey A. Favorite (X-5), Mark A. Fitzgerald (TSA-1), M. A. Gomez (T-12), James E. Gubernatis (T-11), Naomichi Hatano (T-11), Henry Lichtenstein (X-6), Richard R. Picard (TSA-1), Lawrence R. Pratt (T-12), and Richard N. Silver (MSA-11)

\begin{abstract}
For many scientific calculations, Monte Carlo is the only practical method available. Unfortunately, standard Monte Carlo methods converge slowly as the square root of the computer time. We have shown, both numerically and theoretically, that the convergence rate can be increased dramatically if the Monte Carlo algorithm is allowed to adapt based on what it has learned from previous samples. As the learning continues, computational efficiency increases, often geometrically fast. The particle transport work achieved geometric convergence for a two-region problem as well as for problems with rapidly changing nuclear data. The statistics work provided theoretical proof of geometric convergence for continuous transport problems and promising initial results for airborne migration of particles. The statistical physics work applied adaptive methods to a variety of physical problems including the threedimensional Ising glass, quantum scattering, and eigenvalue problems.
\end{abstract}

\section{Background and Research Objectives}

As the inventor of the Monte Carlo method, the Laboratory has a long-standing and major investment in its use and development. Particle transport simulations, for example, have been critical components in accelerator design, reactor development, and radiation and criticality safety. Monte Carlo has been used by Los Alamos for basic research in areas including physics, biology, chemistry, and materials science. The Monte Carlo work of the Los Alamos research community is internationally recognized, and this project represented a cross-disciplinary effort at an important time in the history of the Laboratory, as its roles and missions are being reshaped and its ability to impact science and technology is being tested.

\footnotetext{
*teb@lanl.gov
} 
Three years ago, the statistical theory for adaptive Monte Carlo lagged well behi $Q$ ST I state of implementation. For example, there was considerable empirical simulation evidence for exponential convergence to zero-variance solutions in continuous state problems, but no supporting theory to explain the phenomenon. This lack of statistical theory left open the possibility of "false learning" with no theoretical guidance on how to prevent it. We have derived exponential rate-of- convergence proofs for both discrete ${ }^{1}$ and continuous ${ }^{2}$ simulations and established the conditions required to preclude false learning. These proofs, which assumed a known functional form for zero variance, have provided guidance for adaptive Monte Carlo research. Theoretical extensions to our work are required to continue the quest for exponential convergence to real, complex problems in transport and statistical analyses of data.

\section{Importance to LANL's Science and Technology Base and National R \& D Needs}

Los Alamos has a huge investment in Monte Carlo methods, Monte Carlo computer codes, and experienced Monte Carlo practitioners. The Monte Carlo physics community here is internationally recognized. Los Alamos also has the largest particle transport effort in the world, as well as being responsible for the world's most widely used particle transport code (MCNP). For the last few years, the statistics community at Los Alamos has participated in the particle transport Monte Carlo effort. As a result, our Monte Carlo methods and statistical analysis are ahead of those of the rest of the world. Los Alamos' combined strength in these fields is unmatched. Largely because of this combined strength and the interest in Monte Carlo solutions, the pioneering work on adaptive Monte Carlo algorithms has either been done at Los Alamos or supported by Los Alamos. This project continued Los Alamos dominance in scientific Monte Carlo methods, helped solve much more complex versions of existing problems, and promoted entry into new areas of science in the national interest.

Our research has provided the foundation to achieve an enormous scientific impact. Not only are there many problems that cannot be solved efficiently without new methods, there are also problems that are so difficult that they are not even attempted. In addition, complex experiments are becoming progressively more difficult and costly. Although there must always be a place for these experiments, their cost and complexity will generally remove them from the day-to-day design process. Theoretical projects such as this one become even more important to fill the experimental gap in a cost-effective manner.

Most of Los Alamos' large multidisciplinary programs already rely on Monte Carlo methods for some part of the program. 
The nuclear weapons program, nuclear safety programs, system simulation and war games, materials research, and other physics, chemistry, and biology problems require Monte Carlo solutions. If the project's successes can be extended, then present problems using adaptive Monte Carlo methods could be done in a fraction of the current computer time allowing more detailed and complex computations to be done.

The objectives of this adaptive Monte Carlo methods project were to: (1) examine several recent significant developments in the methods; (2) extend and apply them to key bottleneck problem areas; and (3) develop new methods that would achieve significant improvement in the efficiency of applications of the method.

\section{Scientific Approach and Accomplishments}

Note that this project was terminated after one year in response to reductions in LDRD Program funding. These are the accomplishments for the year in particle transport, statistics, and statistical physics.

The particle transport work concentrated on achieving geometric convergence. The reduced source method achieved geometric convergence on a two-region problem by iteratively using the interface leakage from one region as a source into the other region. ${ }^{3,4} \mathrm{We}$ also achieved geometric convergence for energy-independent transport in a rectangular box, although a (removable) approximation limited the geometric convergence to the first few iterations. $^{5,6}$ Adaptive importance sampling achieved geometric convergence on energydependent problems with rapidly varying cross section and importance functions by allowing the Monte Carlo algorithm to adaptively learn better basis functions.?

The statistical work provided the seminal theoretical proof of geometric convergence for continuous state space problems, ${ }^{2}$ in the process supplying sufficient conditions for avoidance of false learning. Related simulation results on adaptive learning algorithms for airborne migration of particles in homogeneous turbulence showed acceleration in CPU time by factors of tens to hundreds; such results have potential application to pollution modeling and chemical/biological warfare. We also completed a theoretical proof of variance reduction regarding the use of transition dynamics ${ }^{8,9}$ relative to the standard Metropolis analysis for statistical physics problems.

The statistical physics work focused on applying extensions of recently developed algorithms to a variety of physical problems. ${ }^{10}$ We applied a bivariate version of the

multicanonical method to the three-dimensional Ising glass. ${ }^{11,12}$ At low temperatures, we found its ground state to be consistent with the droplet picture. At higher temperatures, but still 
At higher temperatures, but still below the glass transition temperature, we found the ground state to be consistent with the ultrametric picture.

We improved algorithms for quantum scattering and eigenvalue problems and applied them to parity violation in low-energy scattering in nuclear physics. ${ }^{13,14}$. We also developed a new adaptive approach to the construction of trial wave functions for diffusion Monte Carlo methods and applied it to the problem of $\mathrm{D}_{3} \mathrm{O}^{+}$in an aqueous solution. ${ }^{15}$ An improved estimate of the excited state energy resulted.

\section{Publications}

1. Kollman, C., et al., "Adaptive Importance Sampling on Discrete Markov Chains," Annals of Applied Probability 9, 391 (1999).

2. Baggerly, K., et al., "Exponential Convergence of Adaptive Importance Sampling for Markov Chains," (to appear June 2000 in the Journal of Applied Probability).

3. Lichtenstein, H., "Exponential Convergence Rates for Reduced-Source Monte Carlo Transport in $[\mathrm{x}, \mu]$ Geometry," Nucl. Sci. Eng. 133, 258-268 (1999).

4. Lichtenstein, H., "Exponential Convergence for Transport in Spatially PiecewiseConstant $[\mathrm{x}, \mu]$ Geometry," Los Alamos National Laboratory report LA-UR-98-4906, (October 28, 1998).

5. Favorite, J. A., "Exponential Monte Carlo Convergence on a Homogeneous Right Parallelepiped Using the Reduced Source Method with Legendre Expansion," International Conference on Mathematics and Computation, Reactor Physics and Environmental Analysis in Nuclear Applications, Madrid, Spain Sept. 5-10, vol. 1, 283-293 (1999).

6. Favorite, J. A., and H. Lichtenstein, "Exponential Monte Carlo Convergence of a ThreeDimensional Discrete Ordinates Solution," Trans. Am. Nucl. Soc. 81, 147 (1999).

7. Booth, T. E., "Adaptive Importance Sampling with a Rapidly Varying Importance Function," Los Alamos National Laboratory document LA-UR-99-3611 Submitted to Nucl. Sci. Eng.

8. Fitzgerald, M., et al., "Canonical Transition Probabilities for Adaptive Metropolis Simulation," Europhys. Lett. 46, 282 (1999).

9. Fitzgerald, M., et al., "Monte Carlo Transition Dynamics and Variance Reduction," ( to be published in J. Stat. Phys. Jan 2000 issue) .

10. Gubernatis, J. E. and N. Hatano, "The Multi-Canonical Method,". (to be published in Computers in Science and Engineering). 
11. Hatano, N. and J.E. Gubernatis, "A Bi-Variate Multicanonical Monte Carlo Method for the Three-Dimensional \pm J Ising Model," in Computer Simulations Studies in Condensed Matter Physics XII, D. Landau, Ed. (Springer-Verlag, Heidelberg, in press).

12. Hatano, N. and J. E. Gubernatis, "Bi-Variate Multicanonical Monte Carlo Method for the Three-Dimensional \pm J Spin Glass, in Slow Dynamics in Complex Systems," Tokuyama nas I. Oppenheim, Eds. (American Institute of Physics, New York, 1999), p. 565.

13. Carlson, J., "Quantum Monte Carlo Methods in Nuclear Physics," in Quantum Monte Carlo Methods in Physics and Chemistry, M. P. Nightingale and C. J. Umrigar, Eds. (Kluwer, Dordrecht, 1999), p. 287.

14. Carlson, J., "Quantum Monte Carlo in Few Body Systems," Few Body Systems Supplement 10, 1 (1999).

15. Gomez, M. A. and L. R. Pratt, "Construction of Simulation Wave Functions for Aqueous Species: $\mathrm{D}_{3} \mathrm{O}^{+}, " J$. Chem. Phys. 109, 8783 (1999).

16. Booth, T. E., "An Approximate Monte Carlo Adaptive Importance Sampling Method," Los Alamos National Laboratory document LA-UR-00-404 Submitted to Nucl. Sci. Eng.

17. Fitzgerald, M., et al., "Adaptive Monte Carlo in Generalized Ensembles, in Condensed Matter Theories," Vol. 14, D. Ernst, I. Perakis, and S. Umar, Eds. (Nova Science Publishers, Commack, New York, in press).

18. Gomez, M. A. et al., "Molecular Realism in Default Models for Information Theories of Hydrophobic Effects," J. Phys. Chem. 103, 3520 (1999). 